Unfallchirurg 2009 • 112:837

DOI 10.1007/s00113-009-1596-z

(c) Springer Medizin Verlag 2009

\author{
F. Hildebrand · C. Krettek \\ Unfallchirurgische Klinik, Medizinische Hochschule Hannover
}

\title{
Aktuelle Konzepte in der Therapie des polytraumatisierten Patienten
}

Die Unfallchirurgie versteht sich als diejenige medizinische Disziplin, die schwerverletzte Patienten vom Unfallort bis zum Abschluss der Rehabilitation betreut. Somit besteht auch die Verpflichtung, eine stetige Verbesserung der Versorgungsqualität schwerverletzter Patienten zu erreichen.

\section{Traumanetzwerke}

Diesem Aspekt wird durch die Neu- und Weiterentwicklung qualitätssichernder Maßnahmen Rechnung getragen. Hierzu gehört die voranschreitende Etablierung von Traumanetzwerken mit der Zertifizierung der teilnehmenden Kliniken mit dem Ziel, jedem Schwerverletzten in Deutschland rund um die Uhr die bestmögliche Versorgung unter standardisierten Qualitätsmaßstäben zu ermöglichen. Auch die Umsetzung des Netzwerks Katastrophenmedizin zur Entwicklung strukturierter Lösungsvorschläge für die Versorgung großer Verletzten- und Schwerverletztenzahlen ist in diesem $\mathrm{Zu}$ sammenhang ein wesentlicher Faktor. Bei der Bewältigung der Situation eines Massenanfalls an Verletzten sieht sich die Unfallchirurgie als primär zuständige Fachdisziplin und steht somit in einer besonderen Verantwortung.

\section{ATLS-, PTLS- und DSCT-Kurse}

Da die Sicherung der Versorgungsqualität auch in einem direkten Zusammenhang mit der Ausbildung des medizinischen Personals und den Behandlungsstandards steht, ist die Durchführung verschiedener Fortbildungsformate der präklinischen, frühen klinischen und definitiven Versorgung schwerverletzter Patienten von essenzieller Bedeutung. Die Schockraumbehandlung nach dem ATLS-Konzept sowie die angelehnte präklinische Variante (PTLS) dienen der schnellen Einschätzung des Zustands und der prioritätenorientierten Behandlung des schwerverletzten Patienten. Neben diesen etablierten Fortbildungsformaten wurde im November der DSCT-Inaugurationskurs durchgeführt, in dem die Entscheidungsfindung, das Traumamanagement und chirurgische Techniken der operativen Versorgung schwerverletzter Patienten innerhalb der ersten operativen Phase im Anschluss an die Schockraumphase gelehrt werden.

\section{Damage Control Orthopedics}

Ziel einer aktuellen Studie zur Bedeutung der initialen Frakturversorgung bei schwerverletzten Patienten ist die Identifizierung der Risikopopulation, die insbesondere vom Konzept des Damage Control Orthopedics profitieren kann. Hintergrund dieses verletzungsadaptierten Vorgehens ist eine Reduktion der systemischen Entzündungsreaktion, die sich additiv durch das Trauma selbst („first hit") und u. a. dem operationsbedingten Trauma („second hit") zusammensetzt.
Die der posttraumatischen Entzündungsreaktion zu Grunde liegenden pathophysiologischen Veränderung werden im Eröffnungsbeitrag dieses Themenhefts dargestellt.

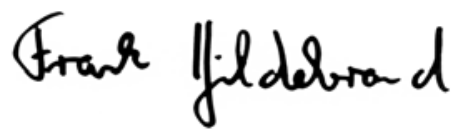

Priv.-Doz. Dr. F. Hildebrand

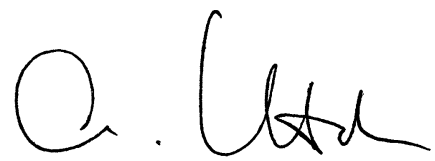

Prof. Dr. C. Krettek, FRCS, FRACS

\section{Korrespondenzadressen PD Dr. F. Hildebrand}

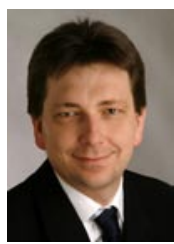

Unfallchirurgische Klinik, Medizinische Hochschule Hannover Carl-Neuberg Straße 1, 30625 Hannover Hildebrand.Frank@ mh-hannover.de

\section{Prof. Dr. C. Krettek}

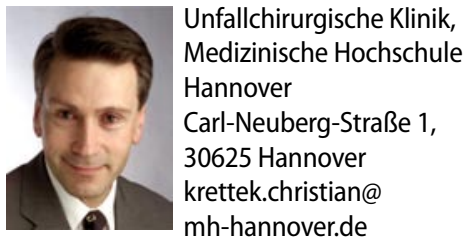

